Punjab University Journal of Mathematics

(ISSN 1016-2526)

Vol. 53(1)(2021) pp. 35-53

\title{
Impact of Homogeneous/Heterogeneous Reactions and Convective Conditions on Peristaltic Fluid Flow in a Symmetric Channel
}

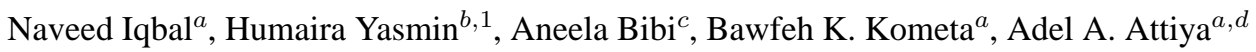

${ }^{a}$ Department of Mathematics, College of Science, University of Ha'il, 81481, Saudi Arabia ${ }^{b}$ Department of Basic Sciences, Deanship of Preparatory Year, King Faisal University, Al-Ahsa, 31982, Saudi Arabia

${ }^{c}$ Department of Mathematics, Quaid-I-Azam University 45320, Islamabad 44000, Pakistan

${ }^{d}$ Department of Mathematics, Faculty of Science, University of Mansoura, Mansoura, 35516, Egypt Email ${ }^{1}$ : hhassain@kfu.edu.sa

Received: 17 September, 2020 / Accepted: 22 December, 2020 / Published online: 08 January, 2021

\begin{abstract}
Simultaneous impacts of homogeneous and heterogeneous reaction and Joule heating in magnetohydrodynamic (MHD) peristaltic flow of viscous fluid in a symmetric channel are analyzed in this investigation. Attention has been focused on designing and simulating a mathematical model for a viscous fluid in presence of viscous dissipation. Long wavelength approximation in wave frame analysis is implemented. Expressions for the stream function, axial pressure gradient, temperature, heat transfer coefficient and concentration are derived and discussed. In addition, the trapping phenomenon is analyzed. The effects of the physical quantities of concern are viewed with a special focus on homogeneous and heterogeneous reaction and convective conditions for the transfer of heat at the walls. It is observed that the pressure rise first increases and then decreases with an increase in amplitude ratio. Effects of Brinkman and Hartmann numbers on temperature are quite analogous and a temperature rise is observed, however, temperature decays for the increased value of Biot number. Moreover, fluid concentration decreases when the value of the homogeneous reaction parameter is increased.
\end{abstract}

AMS (MOS) Subject Classification Codes: 76D05; 76R99; 76Z05; 80A05; 80A32

Key Words: Peristalsis, homogeneous/heterogenous reaction, Joule heating, convective conditions. 


\section{INTRODUCTION}

The principle of peristalsis is usually used in roller and finger pumps, transfer of urine from the kidneys to the urinary bladder, movement of lymph vessels, vasomotion of blood capillaries, ingestion of food through the esophagus and transmitting semen. To prevent pollution of the outside atmosphere, explosive isotopes in the nuclear power industry should be transferred by this mechanism. Such a mechanism concerning the heat transfer emerges for the conduction of heat in the tissues, the convection of heat by the blood circulation from the membranes of the body tissues and the radiation between both the environment and the surface. Peristalsis is implicated by heat transfer in oxygen delivery and blood transfusion processes. Mass transfer is also significant in the molecular diffusion, when nutrients disperse from the blood vessels to the surrounding tissues, diffusion of chemical impurities, combustion process, membrane-separation process, reverse osmosis and distillation process. The magnetohydrodynamic (MHD) concept through ducts/channels has promising applications in the design of shock tubes, MHD generators, pumps and flow meters and cross-field accelerators. The flow in such devices is supplemented by heat either dissipated internally through Joule or viscous heating or that generated by electric currents across the walls. Motivated by all the aforementioned facts, various recent researchers $[1-8]$ have analyzed the heat and mass transfer effects in the peristaltic flows since the primary attempts one by Latham [16] and the other by Shapiro et al. [17]. Hina et al. [9] investigated the peristaltic flow of Powell-Eyring fluid in a curved channel with heat transfer. Ahmed et al. [10] examine simulation results of mixed convective MHD peristaltic flow with joule heating in a curved pipe. Thermo-mechanical analysis of the non-Newtonian peristaltic mechanism by using a modified heat flux model is done by Mehmood et al. [11]. Riaz et al. [12] analyzed the importance of hybrid nanoparticles in the heat transfer of the Eyring-Powell fluid in the peristaltic flow regime. Yasmin et al. [13] emphasized the importance of the mechanism of heat/mass transfer in the peristalsis of the rheological substance. Hayat et al. [14] investigated the effects of magnetic field, thermal radiation and heat/mass convection on peristaltic flow. Yasmin et al. [15] analyzed the heat/mass transfer through convective boundary conditions on a Johnson-Segalman fluid flow with peristalsis in a curved channel.

It is seen from the available information on the subject that there is very little emphasis yet on the impact of the homogeneous and heterogeneous chemical reactions in a flow with peristalsis $[18-21]$. It is noticeable that the homogeneous and heterogeneous processes are important in various thermochemical processes, such as fermentation, electrochemical, catalysis, semiconducting film and biochemical processing. Interpretations of such reactions involve coal gasification, iron manufacturing in blast furnaces and oxidative restoration in coke catalysis, etc.. The rate of conversion of reactant is different in both homogeneous-heterogeneous reactions on the catalytic surface and within the fluid. Motivated by such applications, the present study focuses on the impact of the homogeneous and heterogeneous chemical reactions on peristaltic viscous fluid flow along with MHD, the Joule heating and viscous dissipation. The channel walls satisfy convective conditions. The arrangement of the present attempt is as follows. The next section comprises problem formulation. Solution expressions are presented in section three. Analysis of the numerous 
parameters on important flow quantities is addressed in section four. Section five consists of the main points.

\section{PROBLEM FORMULATION}

The electrically conductive viscous fluid (incompressible) is assumed in a two-dimensional symmetrical channel of width. $2 a$. The channel walls satisfy convective conditions. With constant speed $c$, a wave of amplitude $b$ (which is sinusoidal) is propagating along the walls of the channel in the $\bar{X}$-direction and $\bar{Y}$-axis is taken normal to the channel walls. A uniform magnetic field with magnetic flux density $\bar{B}=\left(0, B_{0}, 0\right)$ is applied and the induced magnetic field is neglected by assuming a small magnetic Reynolds number. The following formulations provide a basic model for homogeneous-heterogeneous reactions [18 - 21] in the presence of the two chemical species $A$ and $B$ :

$A+2 B \rightarrow 3 B$, rate $=k_{c} \alpha \beta^{2}$,

$A \rightarrow B$, rate $=k_{s} \alpha$.

Here $\alpha$ and $\beta$ are the concentrations of the chemical species $A$ and $B$ and $k_{c}$ and $k_{s}$ are the rate constants. We also consider that reactant $A$ has a constant concentration $\alpha_{0}$ and no auto-catalyst $B$ occurs above the channel walls. Consequently, the reaction mechanism guarantees that the reaction rate is zero for the outer flow. The configuration of the walls of the channel is established by

$$
\bar{H}(\bar{X}, \bar{t})=a+b \cos \left(\frac{2 \pi}{\lambda}(\bar{X}-c \vec{t})\right),
$$

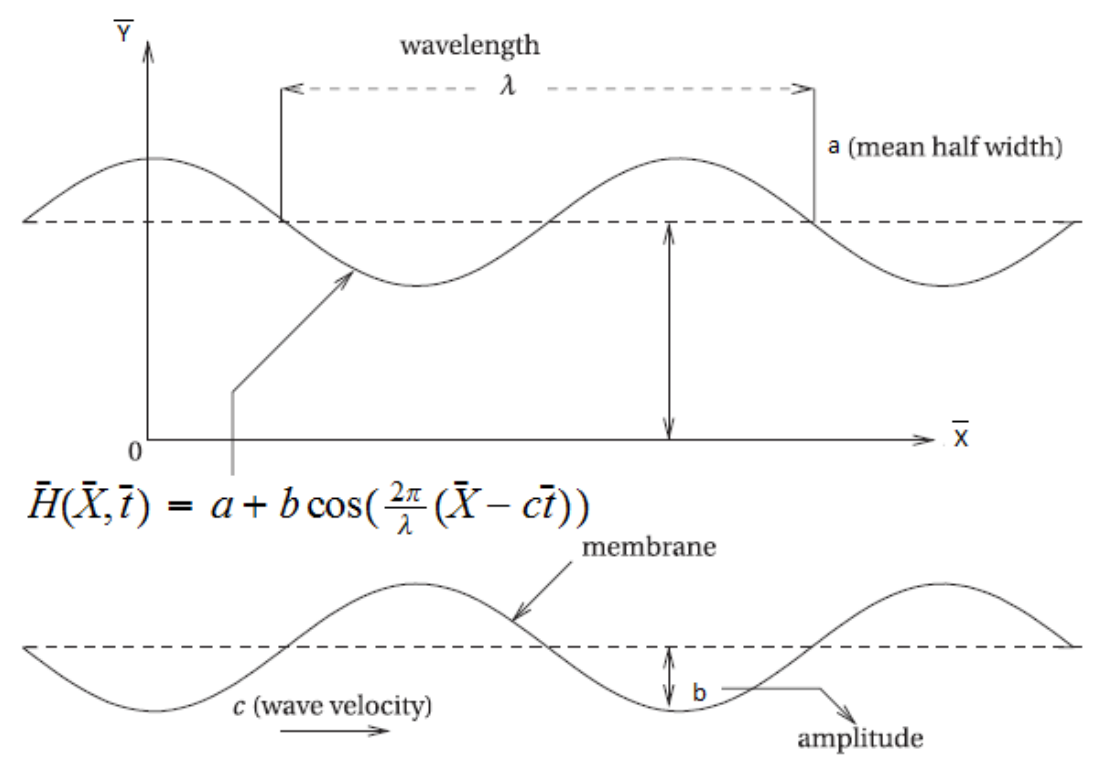

Figure 1: Geometry of the problem 
where the wave amplitude is $b$, the wavelength is $\lambda$ and the time is $\bar{t}$. The equations which regulate the flow of incompressible viscous fluid with MHD with the effects of viscous dissipation and Joule heating in presence of heat as well as mass transfer $[13,14,18]$ are as follows:

$$
\begin{gathered}
\frac{\partial \bar{U}}{\partial \bar{X}}+\frac{\partial \bar{V}}{\partial \bar{Y}}=0, \\
\rho \frac{d \bar{U}}{d \bar{t}}=-\frac{\partial \bar{P}}{\partial \bar{X}}+\mu\left[\frac{\partial^{2} \bar{U}}{\partial \bar{X}^{2}}+\frac{\partial^{2} \bar{U}}{\partial \bar{Y}^{2}}\right]-\sigma B_{0}^{2} \bar{U}, \\
\rho \frac{d \bar{V}}{d \bar{t}}=-\frac{\partial \bar{P}}{\partial \bar{Y}}+\mu\left[\frac{\partial^{2} \bar{V}}{\partial \bar{X}^{2}}+\frac{\partial^{2} \bar{V}}{\partial \bar{Y}^{2}}\right], \\
\rho \xi \frac{d \bar{T}}{d \bar{t}}=\kappa\left[\frac{\partial^{2} \bar{T}}{\partial \bar{X}^{2}}+\frac{\partial^{2} \bar{T}}{\partial \bar{Y}^{2}}\right]+\mu\left[\left(\frac{\partial \bar{U}}{\partial \bar{X}}+\frac{\partial \bar{V}}{\partial \bar{Y}}\right)^{2}+2\left(\frac{\partial \bar{U}}{\partial \bar{X}}\right)^{2}+2\left(\frac{\partial \bar{V}}{\partial \bar{Y}}\right)^{2}\right]+\sigma B_{0}^{2} \bar{U}^{2}, \\
\frac{d \bar{\alpha}}{d \bar{t}}=D_{A}\left[\frac{\partial^{2} \bar{\alpha}}{\partial \bar{X}^{2}}+\frac{\partial^{2} \bar{\alpha}}{\partial \bar{Y}^{2}}\right]-k_{c} \bar{\alpha} \bar{\beta}^{2}, \\
\frac{d \bar{\beta}}{d \bar{t}}=D_{B}\left[\frac{\partial^{2} \bar{\beta}}{\partial \bar{X}^{2}}+\frac{\partial^{2} \bar{\beta}}{\partial \bar{Y}^{2}}\right]+k_{c} \bar{\alpha} \bar{\beta}^{2} .
\end{gathered}
$$

In above equations $\bar{U}, \bar{V}$ represent the fluid velocity components in the $\bar{X}$ and $\bar{Y}$ directions, $\rho$ is the fluid density, $\bar{P}$ denotes the pressure, $\frac{d}{d \bar{t}}\left(=\frac{\partial}{\partial \bar{t}}+\bar{U} \frac{\partial}{\partial \bar{X}}+\bar{V} \frac{\partial}{\partial \bar{Y}}\right)$ is the expression of the material time derivative, $\bar{T}$ is the fluid temperature, $\sigma$ is the electrical conductivity, $\mu$ is the fluid's dynamic viscosity, $\kappa$ is the fluid's thermal conductivity, $\xi$ is the specific heat at constant volume and $D_{A}, D_{B}$ are the coefficients of diffusion for the corresponding chemical species $A$ and $B$.

The boundary conditions relevant to the problem are defined by

$$
\begin{aligned}
\frac{\partial \bar{U}}{\partial \bar{Y}} & =0, \bar{T}=\bar{T}_{0}, \bar{\alpha}=\bar{\alpha}_{0}, \bar{\beta}=0, \text { at } \bar{Y}=0, \\
\bar{U} & =0, k \frac{\partial \bar{T}}{\partial \bar{Y}}=-h_{t}\left(\bar{T}-\bar{T}_{1}\right), D_{A} \frac{\partial \bar{\alpha}}{\partial \bar{Y}}=k_{s} \bar{\alpha}, D_{B} \frac{\partial \bar{\beta}}{\partial \bar{Y}}=-k_{s} \bar{\beta}, \text { at } \bar{Y}=(\overline{\mathcal{H}}, 9)
\end{aligned}
$$

in which $k$ is the thermal conductivity, $h_{t}$ the wall heat transfer coefficient and $\bar{T}_{1}$ the temperature at the wall. No-slip condition is considered for the fluid velocity. Last two conditions are imposed for the concentrations of the two chemical species $A$ and $B$ on the channel wall.

In the case of two-dimensional unsteady flow of viscous fluid, one can write the velocity $\overline{\mathbf{V}}$ in the form

$$
\overline{\mathbf{V}}=(\bar{U}(\bar{X}, \bar{Y}, \bar{t}), \bar{V}(\bar{X}, \bar{Y}, \bar{t}), 0)
$$

Using the transformations

$$
\begin{aligned}
& \bar{x}=\bar{X}-c \bar{t}, \bar{y}=\bar{Y}, \bar{u}(\bar{x}, \bar{y})=\bar{U}(\bar{X}, \bar{Y}, \bar{t})-c, \bar{v}(\bar{x}, \bar{y})=\bar{V}(\bar{X}, \bar{Y}, \bar{t}), \\
& \bar{T}(\bar{x}, \bar{y})=\bar{T}(\bar{X}, \bar{Y}, \bar{t}), \bar{p}(\bar{x}, \bar{y})=\bar{P}(\bar{X}, \bar{Y}, \bar{t}) .
\end{aligned}
$$


In the moving/wave frame, the unsteady motion $(\bar{X}, \bar{Y}, \bar{t})$ becomes steady $(\bar{x}, \bar{y})$ in the stationary/fixed frame. Here $(\bar{u}, \bar{v})$ are components of the fluid velocity in the moving/wave frame and the pressure is $\bar{p}$.

Introducing

$$
\begin{aligned}
& x=\frac{\bar{x}}{\lambda}, y=\frac{\bar{y}}{a}, u=\frac{\bar{u}}{c}, v=\frac{\bar{v}}{c \delta}, p=\frac{a^{2} \bar{p}}{c \mu \lambda}, \delta=\frac{a}{\lambda}, \Re=\frac{\rho c a}{\mu}, \\
& v=\frac{\mu}{\rho}, M=\sqrt{\left(\frac{\sigma}{\mu}\right)}, \operatorname{Pr}=\frac{\mu \xi}{k}, E=\frac{c^{2}}{\xi\left(\bar{T}_{1}-\bar{T}_{0}\right)}, B r=\operatorname{Pr} E, h=\frac{\bar{h}}{a}, \\
& d=\frac{b}{a}, t=\frac{c \bar{t}}{\lambda}, \theta=\frac{\bar{T}-\bar{T}_{0}}{\bar{T}_{1}-\bar{T}_{0}}, \bar{\alpha}=\frac{f}{\alpha_{0}}, \bar{\beta}=\frac{g}{\alpha_{0}}
\end{aligned}
$$

and defining $u$ and $v$ (i.e., the fluid velocity components) in form of the stream function $\psi$ by

$$
u=\frac{\partial \psi}{\partial y}, v=-\frac{\partial \psi}{\partial x}
$$

the condition of incompressibility (2) is satisfied identically and Eqs. $(3-7)$ through low Reynolds number and long wave-length yield

$$
\begin{aligned}
\frac{d p}{d x}=\frac{\partial^{3} \psi}{\partial y^{3}}-M^{2}\left(\frac{\partial \psi}{\partial y}+1\right), & \frac{\partial^{4} \psi}{\partial y^{4}}-M^{2} \frac{\partial^{2} \psi}{\partial y^{2}} \\
\frac{\partial p}{\partial y} & =0, \\
\frac{\partial^{2} \theta}{\partial y^{2}}+B r\left(\frac{\partial^{2} \psi}{\partial y^{2}}\right)^{2}+M^{2} B r\left(\frac{\partial \psi}{\partial y}+1\right)^{2} & =0, \\
\frac{1}{S c} \frac{\partial^{2} f}{\partial y^{2}}-K f g^{2} & =0, \\
\frac{\gamma}{S c} \frac{\partial^{2} g}{\partial y^{2}}+K f g^{2} & =0,
\end{aligned}
$$

where $p \neq p(y), v$ represents the fluid kinematic viscosity, $M$ is the Hartmann number, $\mathrm{Br}$ is the Brinkman number, $\mathrm{Pr}$ is the Prandtl number, the Eckert number is denoted by $E$, the wave number by $\delta$, Schmidt number is symbolized as $S c, K=\frac{a^{2} k_{c} \alpha_{0}^{2}}{v}$ is the homogenous reaction strength, $\gamma=\frac{D_{B}}{D_{A}}$ denotes the diffusion coefficients ratio, $\theta(y)$ is the fluid temperature in dimensionless form and $f, g$ are the chemical species concentrations in the dimensionless form respectively after dropping bars. 
The dimensionless boundary conditions are

$$
\begin{aligned}
\theta & =0, f=1, g=0, \quad \text { at } y=0, \\
\frac{\partial \theta}{\partial y} & =-B i(\theta-1), \frac{\partial f}{\partial y}=\Gamma f, \gamma \frac{\partial g}{\partial y}=-\Gamma f, \quad \text { at } y=h, \\
\psi & =0, \frac{\partial^{2} \psi}{\partial y^{2}}=0, \quad \text { at } y=0, \\
\psi & =F, \frac{\partial \psi}{\partial y}=-1, \quad \text { at } y=h(x),
\end{aligned}
$$

where $B i=\frac{h_{t} k}{a}$ is the Biot number, $\Gamma=\frac{k_{s} a}{D_{A}}$ measures the strength of the heterogenous (surface) reaction.

The instantaneous volumetric flow rate $Q$ in the stationary/fixed frame is defined by

$$
Q=\int_{0}^{\bar{H}(\bar{X}, \bar{t})} \bar{U}(\bar{X}, \bar{Y}, \bar{t}) .
$$

The expression given above in the wave frame is written as

$$
q=\int_{0}^{\bar{h}(\bar{x})} \bar{u}(\bar{x}, \bar{y}) d \bar{y} .
$$

Using Eqs. (11), (23) and (24), we can constitute the following expression:

$$
Q=q+c \bar{h}(\bar{x})
$$

Time-mean flow over a period $\tau(=\lambda / c)$ is specified as

$$
\bar{Q}=\frac{1}{\tau} \int_{0}^{\tau} Q d \bar{t} .
$$

Putting Eq. (25) into Eq. (26) and then integration of the resulting expression gives

$$
\bar{Q}=q+c a \text {. }
$$

In the laboratory and the wave frames, the dimensional time-mean flows $\eta$ and $F$ can be placed into the forms

and then (29) can be reduced as follows:

$$
\eta=\frac{\bar{Q}}{c a}, F=\frac{q}{c a}
$$

$$
\eta=F+1
$$

$$
F=\int_{0}^{h(x)} \frac{\partial \psi}{\partial y} d y=\psi(h(x))-\psi(0) .
$$

The dimensionless form of $h$ is

$$
h(x)=1+d \cos (2 \pi x),
$$

where $d=\frac{b}{a}$. The pressure rise in dimensionless form is given by

$$
\Delta p_{\lambda}=\int_{0}^{\lambda} \frac{d p}{d x} d x
$$

where $\frac{d p}{d x}$ is defined in Eq. (14). 
It is estimated that the diffusion coefficients of chemical species $A$ and $B$ are of equivalent size. Therefore $D_{A}=D_{B}$, i.e., $\gamma=1$. This assumption leads to the following relation:

$$
f+g=1 \text {. }
$$

Hence Eqs. (18) and (19) give

$$
\frac{1}{S c} \frac{\partial^{2} f}{\partial y^{2}}-K f(1-f)^{2}=0
$$

and the boundary conditions take the form

$$
f=1, \text { at } y=0 ; \frac{\partial f}{\partial y}=\Gamma f \text {, at } y=h .
$$

\section{Solutions}

The closed form solutions of Eqs. (14), (15) and (17) subject to the corresponding boundary conditions are as follows:

$$
\begin{aligned}
\psi(y)= & \frac{2 e^{h M}(F M y \cosh [h M]+y \sinh [h M]-(F+h) \sinh [M y])}{1+h M+e^{2 h M}(-1+h M)} \\
\frac{d p}{d x}= & \frac{-(F+h) M^{3} \cosh [h M]}{h M \cosh [h M]-\sinh [h M]} \\
\theta(y)= & \frac{1}{2\left(1+h M+e^{2 h M}(-1+h M)\right)^{2}}\left[-B r e^{-2 M y}(F+h)^{2} M^{2}\left\{e^{2 h M}-4 e^{M(h+y)}-4 e^{3 M(h+y)}\right.\right. \\
& -4 e^{M(3 h+y)}+e^{2 M(h+2 y)}-4 e^{M(h+3 y)}+e^{2 M y} M^{2} y^{2}+2 e^{2 M(h+y)} M^{2} y^{2} \\
& \left.+e^{2 M(2 h+y)} M^{2} y^{2}\right\}-2 B r e^{2 h M}(F+h)^{2} M^{2}(-1+8 \cosh [h M]) \\
& +\frac{1}{(1+B i h)} y\left\{2 B r\left(1+e^{2 h M}\right)(F+h)^{2} M^{3}\left(1+h M+e^{2 h M}(-1+h M)\right)\right. \\
& +B i e^{2 h M}\left(-2+M^{2}\left(2 h^{2}+B r(F+h)^{2}\left(-5+h^{2} M^{2}\right)\right)+8 B r(F+h)^{2} M^{2} \cosh (h M)\right. \\
& \left.\left.+\left(2+M^{2}\left(2 h^{2}+B r(F+h)^{2}\left(-3+h^{2} M^{2}\right)\right)\right) \cosh (2 h M)-4 h M \sinh (2 h M)\right\}\right]
\end{aligned}
$$

The heat transfer coefficient is defined by

$$
\begin{aligned}
Z(x)= & h_{x} \theta_{y}(h), \\
Z(x)= & \frac{1}{\left((1+B i h)\left(1+h M+e^{2 h M}(-1+h M)\right)^{2}\right)} \times\left(B i d \left(-2+3 B r F^{2} M^{2}\right.\right. \\
& -8 B r e^{h M}(F+h)^{2} M^{2}-8 B r e^{3 h M}(F+h)^{2} M^{2}+B r h^{4} M^{4} \\
& +2 B r h^{3} M^{3}(1+F M)+2 h M(-2+B r F M(3+F M) \\
& +h^{2} M^{2}\left(-2+B r\left(3+4 F M+F^{2} M^{2}\right)+2 e^{2 h M}\left(2-2 h^{2} M^{2}+B r(F+h(3) .) \text { y }\right)(9)\right.
\end{aligned}
$$

The subsequent Eq. (34) is not linear in $f$. The closed form of the solution is not achievable with the specific values of the parameters being used in this equation. Therefore, the perturbation approach has been used to find a solution for $K$ (a small parameter). Therefore, series expansion of $f$ is given in the form

$$
f=f_{0}+K f_{1}+K^{2} f_{2}+\ldots
$$


Substitution of above equation into Eq. (34) and then arranging in terms of the like powers of $K$, one can get:

\subsection{System of $\mathbf{O}(\mathbf{K})^{0}$ and its solution.}

$$
\begin{gathered}
\frac{\partial^{2} f_{0}}{\partial y^{2}}=0, \\
f_{0}=1, \text { at } y=0, \\
\frac{\partial f_{0}}{\partial y}=\Gamma f_{0}, \text { at } y=h(x) .
\end{gathered}
$$

The Eq. (42) with corresponding boundary condition (43) gives the solution as follows:

$$
f_{0}=1+\frac{y \Gamma}{1-h \Gamma} .
$$

\subsection{System of $\mathbf{O}(\mathbf{K})^{1}$ and its solution.}

$$
\begin{aligned}
& \frac{1}{S c} \frac{\partial^{2} f_{1}}{\partial y^{2}}-f_{0}\left(1-f_{0}\right)^{2}=0, \\
& f_{1}=0, \text { at } y=0, \\
& \frac{\partial f_{1}}{\partial y}=\Gamma f_{1}, \text { at } y=h(x) .
\end{aligned}
$$

Supplementing the Eq. (41) to Eqs. (34) and (35), resolve the obtained equations and then implement the relevant boundary conditions, the solution for $f_{1}$ is as follows:

$$
f_{1}=\frac{S c \Gamma^{2}\left\{y^{4}(-1+h \Gamma)(-5+5 h \Gamma-3 y \Gamma)-2 h^{3} y\left(10-5 h \Gamma+h^{2} \Gamma^{2}\right)\right\}}{60\left(-1+h \Gamma^{4}\right)} .
$$

\subsection{System of $\mathbf{O}(\mathbf{K})^{2}$ and its solution.}

$$
\begin{aligned}
& \frac{1}{S c} \frac{\partial^{2} f_{2}}{\partial y^{2}}-f_{1}-3 f_{0}^{2} f_{1}+4 f_{0} f_{1}=0, \\
& f_{2}=0, \text { at } y=0 \\
& \frac{\partial f_{2}}{\partial y}=\Gamma f_{2}, \text { at } y=h(x) .
\end{aligned}
$$

The solution of above system is

$$
\begin{aligned}
f_{2}= & \frac{1}{25200(-1+h \Gamma)^{7}} S c^{2} \Gamma^{3}\left[-h^{6} y\left(4900-2560 h \Gamma+855 h^{2} \Gamma^{2}-190 h^{3} \Gamma^{3}+19 h^{4} \Gamma^{4}\right)\right. \\
& +420(-1+h \Gamma)\left\{\frac{-1}{8} y^{9} \Gamma^{2}(-1+h \Gamma)+\frac{3}{8} y^{8} \Gamma(-1+h \Gamma)^{2}\right. \\
& -\frac{5}{21} y^{7}(-1+h \Gamma)^{3}-\frac{3}{10} h^{3} y^{5} \Gamma\left(10-5 h \Gamma+h^{2} \Gamma^{2}\right) \\
& \left.\left.+\frac{1}{3} h^{3} y^{4}\left(-10+15 h \Gamma-6 h^{2} \Gamma^{2}+h^{3} \Gamma^{3}\right)\right\}\right] .
\end{aligned}
$$




\section{Discussion}

The purpose of this section is to examine the influence of influential flow parameters on the pumping characteristic, temperature, concentrations, streamlines and heat transfer coefficient.

Now we first examine the nature of the pressure gradient $\frac{d p}{d x}$ and the pressure rise $\Delta P_{\lambda}$ per wavelength $\lambda$ for the various parameter values in the problem under consideration. We noted that the interpretation of the pressure rise requires the integration of $\frac{d p}{d x}$. The analytical solution of this integral is not possible. The numerical integration is then used to determine this integral. The variations in pressure gradient $\frac{d p}{d x}$ for various embedded parameters are illustrated in the Figures $2 \& 3$. The variation in $\frac{d p}{d x}$ for distinct values of Hartmann number is demonstrated in Figure 2 when $d=0.3$ and $\eta=1.4$. It is witnessed that the pressure gradient decreases with an enhancement in $M$. Figure 3 shows the impact of the amplitude ratio $d$ on $\frac{d p}{d x}$ when $M=2$ and $\eta=1$.4. It is observed that the pressure gradient decays near the channel walls while it enhances in the center of the channel. Figure 4 indicates the difference in the pressure rise $\Delta p_{\lambda}$ with the flow rate $\eta$ for the numerous values of amplitude ratio $d$ values when $M=0.5$. We observe that pumping increases in the interval $(-0.5 \leq \eta<0.71)$ and it decreases in the interval $(0.71 \leq \eta<1.5)$. Figure 5 is sketched to see the nature of the pressure rise $\Delta p_{\lambda}$, against flow rate $\eta$ for various values of Hartmann number $M$ when $d=0.3$. It is depicted that the pressure rise increases when Hartmann number $M$ is increased in the interval $(-0.5 \leq \eta<0.1)$ and it decreases in the interval $(0.1 \leq \eta<1.5)$.

The influence of various parameters on the fluid temperature $\theta(y)$ is examined in Figures 6-8. The effect of Hartmann number $M$ is portrayed in Figure 6 when $d=0.3, x=0$, $B i=2, B r=0.5, \eta=1.4$. It is noted that the temperature $\theta(y)$ increases by increasing Hartmann number $M$ due to viscous heating resulted from Joule heating. Figure 7 indicates that the fluid temperature $\theta(y)$ enhances with a rise in the Brinkman number $B r$ when $d=0.3, x=0, B i=2, M=1, \eta=1.4$. Actually, Brinkman number $B r$ represents the viscous dissipation effects. It is very clear that in the flow of viscous fluid, the fluid viscosity extracts the kinetic energy out of the movement of the liquid and converts it into intrinsic energy and hence heats the fluid. Figure 8 shows that the fluid temperature decreases when Biot number $B i$ is increased (where $d=0.3, x=0, B r=0.5, M=1$, $\eta=1.4$ ) as the Biot number is the ratio of resistance to internal heat flow to the resistance to the external heat flow. High values of the Biot number indicate that the convection is the main heat transfer mechanism in the presented study.

The variation in concentration profile and concentration at the surface for different physical parameters are depicted in Figures 9-13. Figure 9 represents that the concentration profile tends to increase with an enhancement in heterogeneous reaction parameter $\Gamma$ when $x=0.1, d=0.3, S c=0.5, K=0.2$. Figure 10 illustrates the effect of the strength of homogenous reaction parameter $K$ on the concentration when $x=0.1, d=0.3, S c=0.5$, $\Gamma=2$. It is clear that $f(y)$ decreases by increasing $K$. Figure 11 indicates the effects of the Schmidt number $S c$ on concentration where $x=0.1, d=0.3, K=0.4, \Gamma=2$. It is noticed that $f$ is decreased by increasing $S c$ as the Schmidt number is the ratio of momentum to the mass diffusivity. The variation in concentration profile is revealed as a function of $h$ for some values of $\Gamma$ in Figure 12 with $x=0.1, d=0.3, S c=0.5$. It is observed that 
concentration increases for larger $\Gamma$. From Figure 13 we see that larger Schmidt number $S c$ decreases the concentration profile $f(y)$ where $x=0.1, d=0.3, \Gamma=2$.

The behavior of heat transfer coefficient $Z(x)$ for different values of embedded parameters is shown in Figures 14-16. The heat transfer coefficient exhibits behavioral oscillations which are expected because of the propagation of peristalsis waves. Figure 14 represents that the magnitude of the coefficient of heat transfer $Z(x)$ increases with a rise in the value of Hartmann number $M$ when $d=0.3, B i=1, B r=0.5, \eta=1.4$. Figure 15 depicts that heat transfer coefficient $Z(x)$ enhances by an increase in the value of Brinkman number $B r$ when $d=0.3, B i=1, M=1, \eta=1.4$. Similar behavior is observed for Biot number $B i$ (see Figure 16) i.e., by increasing the value of Biot number, the magnitude of heat transfer coefficient increases when $d=0.3, B r=0.5, M=1, \eta=1.4$.

The trapping phenomenon is fundamentally the development of a bolus of fluid that circulates internally by closed streamlines. To observe this phenomenon, Figures 17 and 18 are plotted. The streamlines for various Hartmann number $M$ values are shown in Figure 17 when the other parameters are kept constant i.e., $d=0.5, \eta=1.4$. This Figure shows that the size of the trapping bolus decreases while the number of streamlines increases when $M$ increases. Figure 18 demonstrates the trapping pattern for various mean flow rate values $\eta$ when $d=0.5, M=1$. It is noted that the number of streamlines increases and the trapped bolus size decreases when mean flow rate $\eta$ increases. 


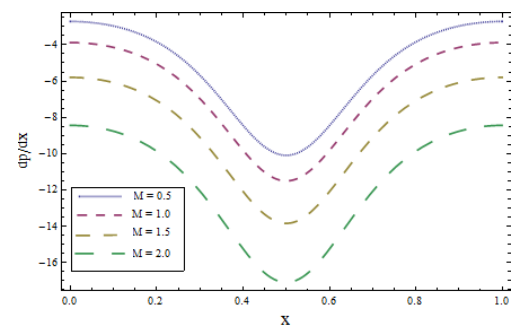

Figure 2: Pressure gradient $\frac{d p}{d x}$ for $M$.

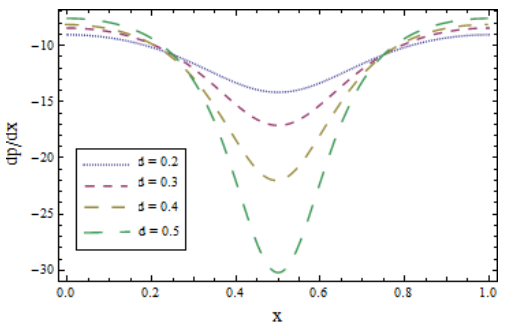

Figure 3: Pressure gradient $\frac{d p}{d x}$ for $d$.

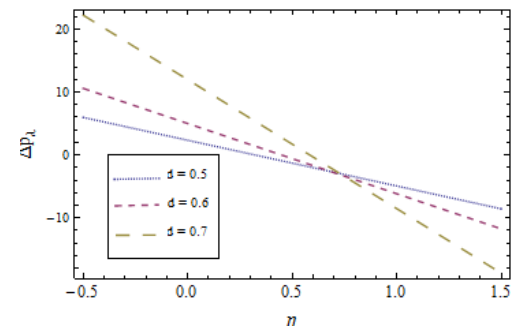

Figure 4: Pressure rise $\Delta P_{\lambda}$ against flow rate for $d$. 


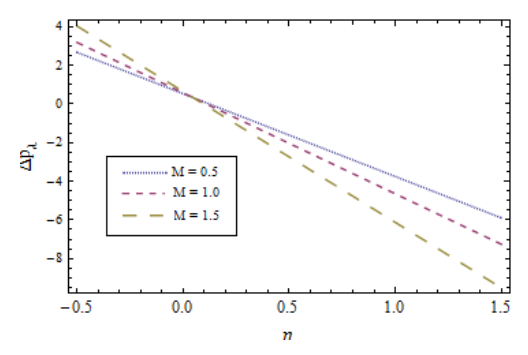

Figure 5: Pressure rise $\Delta P_{\lambda}$ against flow rate for $M$.

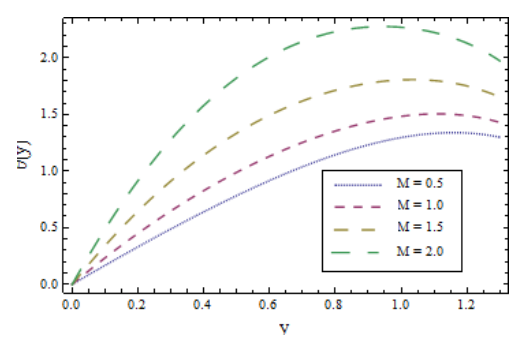

Figure 6: Temperature profile $\theta(y)$ for $M$.

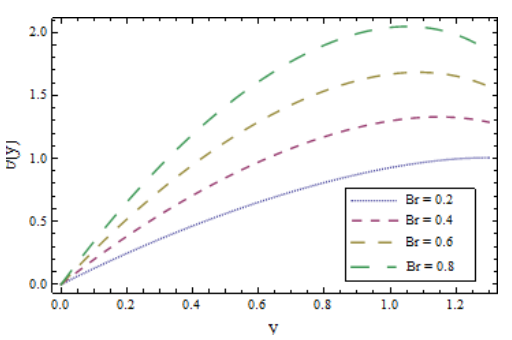

Figure 7: Temperature profile $\theta(y)$ for $B r$. 


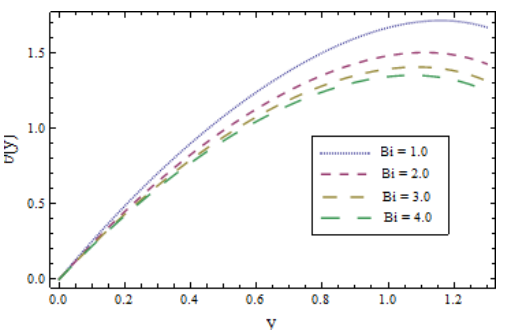

Figure 8: Temperature profile $\theta(y)$ for $B i$.

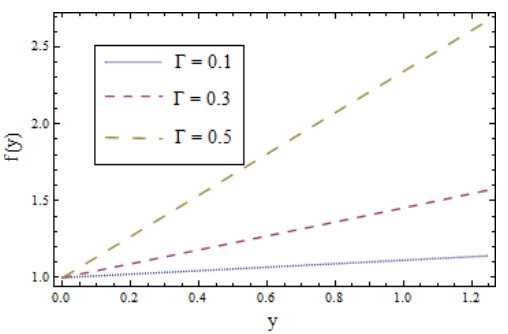

Figure 9: Concentration profile $f(y)$ for $\Gamma$.

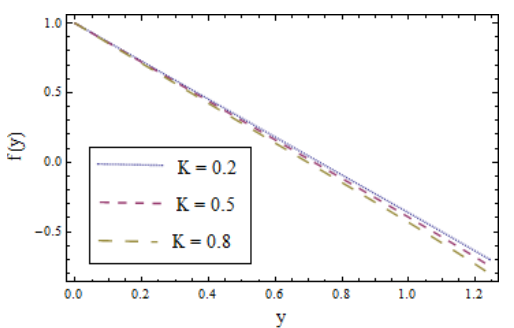

Figure 10: Concentration profile $f(y)$ for $K$. 


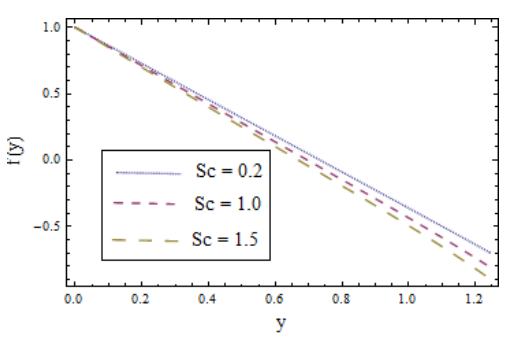

Figure 11: Concentration profile $f(y)$ for $S c$.

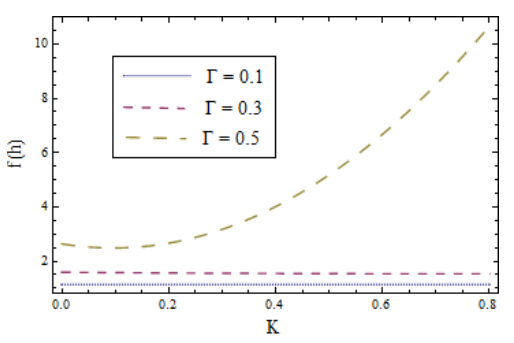

Figure 12: Concentration profile $f(h)$ for $\Gamma$.

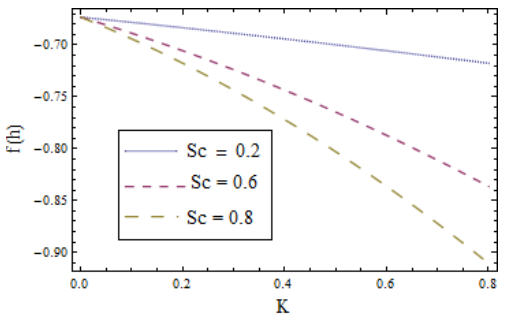

Figure 13: Concentration profile $f(h)$ for $S c$. 


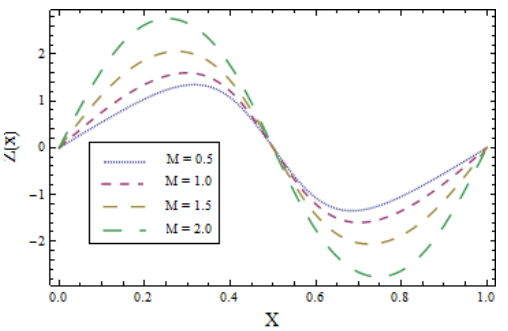

Figure 14: $Z(x)$ for $M$.

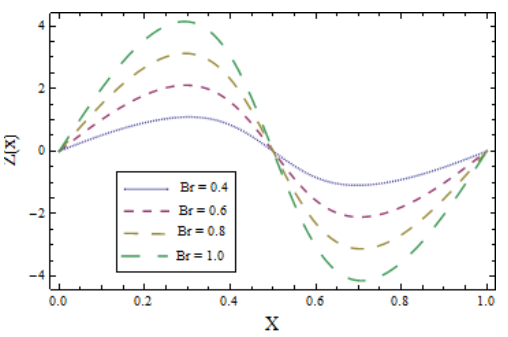

Figure 15: $Z(x)$ for $B r$.

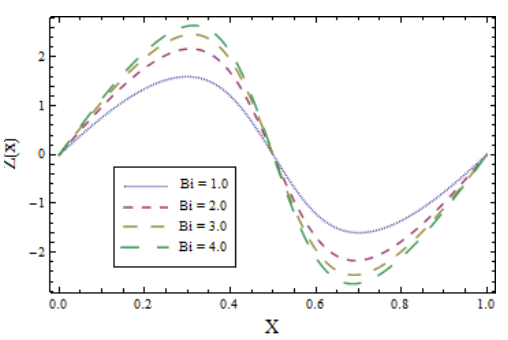

Figure 16: $Z(x)$ for $B i$. 

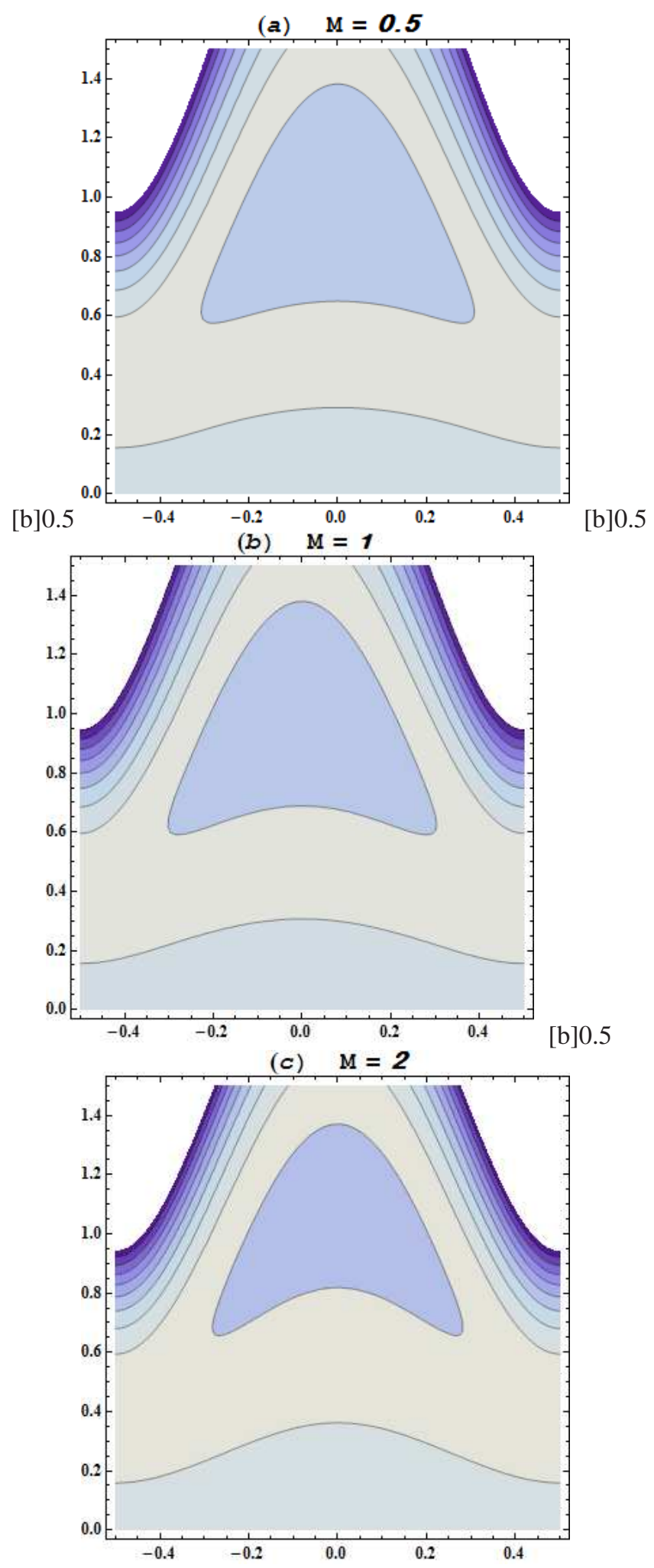

Figure 17: Streamlines for $M$ (panels a-c). 
(a) $\quad \eta=1$
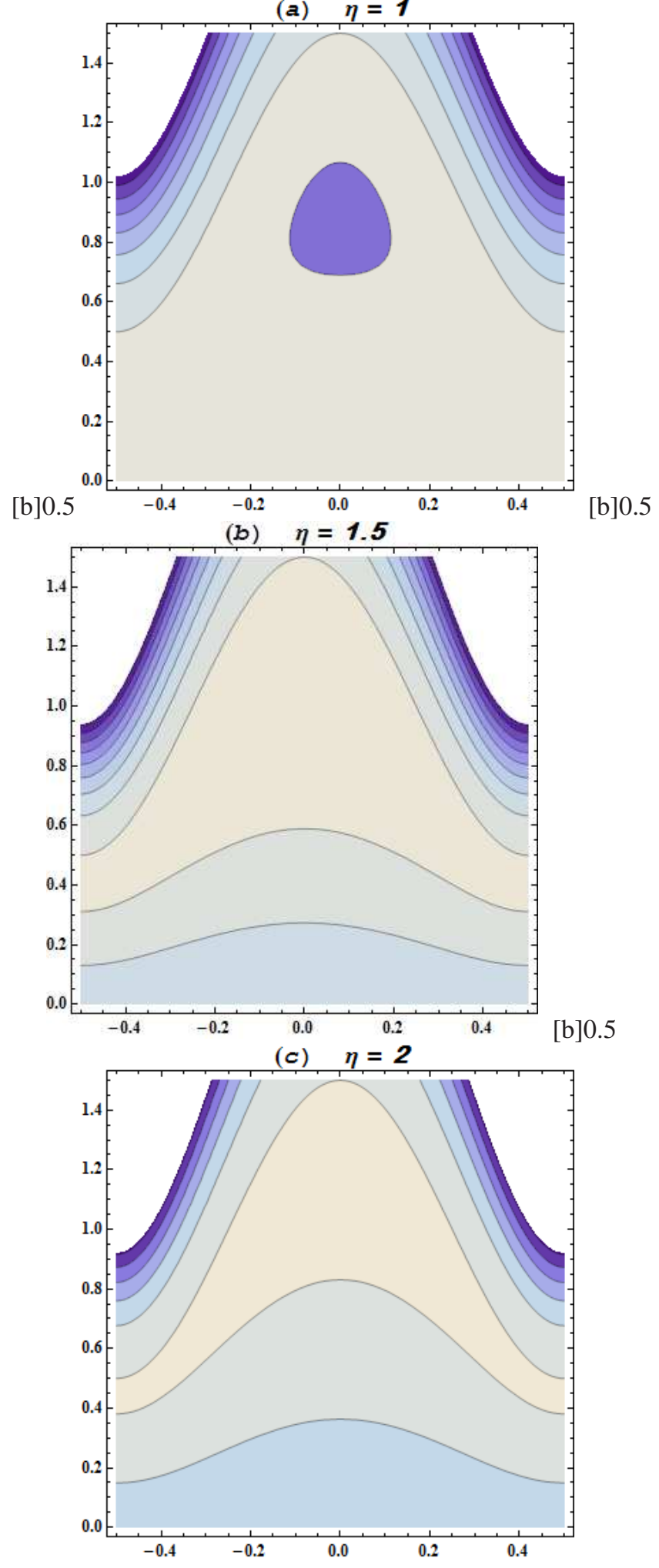

Figure 18: Streamlines for $\eta$ (panels a-c). 


\section{CONCLUDING REMARKS}

Here the impacts of homogeneous and heterogeneous chemical reactions, viscous dissipation, convective conditions and Joule heating on the peristaltic flow in a channel are studied. The presented analysis leads to the following main results.

- The pressure rise first increases and then decreases with an increase in amplitude ratio.

- Effects of Brinkman and Hartmann numbers on temperature are quite analogous i.e., when such parameters increase, the temperature goes up.

- Fluid concentration decreases when the value of homogeneous reaction parameter $K$ is increased.

- Temperature is declined for rise in Biot number.

- Reverse behavior of trapping is depicted for Hartmann number and volume flow rate.

- Concentration of the fluid increases as the heterogeneous reaction parameter $\Gamma$ enhances.

Acknowledgments: This research has been funded by Scientific Research Deanship at University of Ha'il, Saudi Arabia through project number RG-191323.

\section{REFERENCES}

[1] R. Ahmed, N. Ali, S. Ullah Khan and I. Tlili, Numerical simulations for mixed convective hydromagnetic peristaltic flow in a curved channel with joule heating features, AIP Advances 10 (2020) 075303.

[2] M. M. Bhatti , A. F. Elelamy , S. M. Sait and R. Ellahi, Hydrodynamics interactions of metachronal waves on particulate-liquid motion through a ciliated annulus: Application of bio-engineering in blood clotting and endoscopy, Symmetry 14 (2020) 532.

[3] D. S. Bhandari, D. Tripathi and V. K. Narla, Pumping flow model for couple stress fluids with a propagative membrane contraction, Int. J. Mech. Sci., 188 (2020) 105949.

[4] T. Hayat, A. Bibi, H. Yasmin and B. Ahmad, Simultaneous effects of Hall current and homogeneous/heterogeneous reactions on peristalsis, J. Taiwan Inst. Chem. Eng., 58 (2016) 28-38.

[5] T. Hayat, A. Tanveer, H. Yasmin, A. Alsaedi, Homogeneous-heterogeneous reactions in peristaltic flow with convective conditions, Plos One 9 (2014) e113851.

[6] T. Hayat, S. Asghar, A. Tanveer and A. Alsaedi, Homogeneous-heterogeneous reactions in peristaltic flow of Prandtl fluid with thermal radiation, J. Mol. Liq., 240 (2017) 504-513.

[7] T. Hayat, A. Bibi, H. Yasmin, F. E. Alsaadi, Magnetic field and thermal radiation effects in peristaltic flow with heat and mass convection, J. Ther. Sci. Eng. Appli., 10 (2018) 051018.

[8] S. Hina, M. Mustafa, T. Hayat and A. Alsaedi, Peristaltic flow of Powell-Eyring fluid in curved channel with heat transfer: A useful application in biomedicine, Comp. Meth. Prog. Biomed., 135 (2016) 89-100.

[9] M. Javed, A mathematical framework for peristaltic mechanism of non-Newtonian fluid in an elastic heated channel with Hall effect, Multidis. Model. Mat. Struc., (2020) DOI: https://doi.org/10.1108/MMMS-112019-0200.

[10] T. W. Latham, Fluid motion in peristaltic pump (MS thesis), MIT, Cambridge, MA, (1966).

[11] O. U. Mehmood, A. A. Qureshi, H. Yasmin and S. Uddin, Thermo-mechanical analysis of non-Newtonian peristaltic mechanism: Modified heat flux model, Physica A: Statis. Mech. Appli., 550 (2020) 124014.

[12] K. Ramesh, Effects of slip and convective conditions on the peristaltic flow of couple stress fluid in an asymmetric channel through porous medium, Comput. Meth. Prog. Biomedicine, 135 (2016) 1-14.

[13] K. Ramesh, D. Tripathi, M. M. Bhatti and C. M. Khalique, Electroosmotic flow of hydromagnetic dusty viscoelastic fluids in a microchannel propagated by peristalsis, J. Mol. Liq., 314 (2020) 113568.

[14] A. Riaz, R. Ellahi and S. M. Sait, Role of hybrid nanoparticles in thermal performance of peristaltic flow of Eyring-Powell fluid model, J. Them. Anal. Calorim., (2020) DOI: https://doi.org/10.1007/s10973-02009872-9 
[15] A. H. Shapiro, M. Y. Jaffrin and S. L. Weinberg, Peristaltic pumping with long wavelengths at low Reynolds number, J. Fluid Mech., 37 (1969) 799-825.

[16] S. A. Shehzad, F. M. Abbasi, T. Hayat and Fuad Alsaadi, Model and comparative study for peristaltic transport of water based nanofluids, J. Mol. Liquids 209 (2015) 723-728.

[17] G. C. Shit and M. Roy, Hydromagnetic effect on inclined peristaltic flow of a couple stress fluid, Alex. Eng. J., 4 (2014) 949-958.

[18] H. Tariq, A. A. Khan and A. Zaman, Theoretical analysis of peristaltic viscous fluid with inhomogeneous dust particles, Arab. J. Sci. Eng., (2020) DOI: https://doi.org/10.1007/s13369-020-04668-3.

[19] A. Tanveer, T. Hayat, A. Alsaedi and B. Ahmad, Mixed convective peristaltic flow of Sisko fluid in curved channel with homogeneous-heterogeneous reaction effects, J. Mol. Liq., 133 (2017) 131-138.

[20] H. Yasmin, S. Farooq, M. Awais, A. Alsaedi and T. Hayat, Significance of heat and mass process in peristalsis of a rheological material, Heat Trans. Res., 50 (2019) 1561-1580.

[21] H. Yasmin, N. Iqbal and A. Hussain, Convective heat/mass transfer analysis on Johnson-Segalman fluid in a symmetric curved channel with peristalsis: Engineering applications, Symmetry 12 (2020) 1475. 